\title{
Irreducible Equivalence Relations, Gleason Spaces, and de Vries Duality
}

\author{
Guram Bezhanishvili ${ }^{1} \cdot$ Nick Bezhanishvili $^{2}$. \\ Sumit Sourabh ${ }^{2} \cdot$ Yde Venema ${ }^{2}$
}

Received: 17 December 2014 / Accepted: 14 March 2016 / Published online: 20 April 2016

(C) The Author(s) 2016. This article is published with open access at Springerlink.com

\begin{abstract}
By de Vries duality, the category of compact Hausdorff spaces is dually equivalent to the category of de Vries algebras (complete Boolean algebras endowed with a proximity-like relation). We provide an alternative "modal-like" duality by introducing the concept of a Gleason space, which is a pair $(X, R)$, where $X$ is an extremally disconnected compact Hausdorff space and $R$ is an irreducible equivalence relation on $X$. Our main result states that the category of Gleason spaces is equivalent to the category of compact Hausdorff spaces, and is dually equivalent to the category of de Vries algebras.
\end{abstract}

Keywords Compact Hausdorff space - Stone space - Extremally disconnected space · Gleason cover $\cdot$ Boolean algebra $\cdot$ Complete Boolean algebra $\cdot$ Modal algebra $\cdot$ Proximity

Mathematics Subject Classification (2010) 03B45 · 54E05 · 06E15 · 54G05

Nick Bezhanishvili

N.Bezhanishvili@uva.nl

Guram Bezhanishvili

guram@math.nmsu.edu

Sumit Sourabh

sumit.sourabh@gmail.com

Yde Venema

Y.Venema@uva.nl

1 Department of Mathematical Sciences, New Mexico State University, Las Cruces, NM 88003, USA

2 Institute for Logic, Language and Computation, University of Amsterdam, P.O. BOX 94242, 1090 GE Amsterdam, The Netherlands 


\section{Introduction}

By the celebrated Stone duality [29], the category of Boolean algebras and Boolean homomorphisms is dually equivalent to the category of Stone spaces (compact Hausdorff zero-dimensional spaces) and continuous maps. De Vries [14] generalized Stone duality to the category of compact Hausdorff spaces and continuous maps. Objects of the dual category are complete Boolean algebras $B$ with a binary relation $\prec$ (called by de Vries a compingent relation) satisfying certain conditions that resemble the definition of a proximity on a set [26].

Another extension of Stone duality is central to modal logic. We recall that modal algebras are Boolean algebras $B$ with a unary function $\square: B \rightarrow B$ preserving finite meets, and modal spaces (descriptive frames) are Stone spaces $X$ with a binary relation $R$ satisfying certain conditions. Stone duality then generalizes to a duality between the categories of modal algebras and modal spaces (see, e.g., $[11,12,24])$. The dual of a modal algebra $(B, \square)$ is the modal space $(X, R)$, where $X$ is the Stone dual of $B$ (the space of ultrafilters of $B$ ), while the binary relation $R \subseteq X \times X$ is the Jónsson-Tarski dual of $\square$ [23]. Unlike the modal case, in de Vries duality we do not split the dual space of $(B, \prec)$ in two components, the Stone dual of $B$ and the relation $R$. Instead we work with the space of " $\prec$-closed" filters which are maximal with this property.

The aim of this paper is to develop an alternative "modal-like" duality for de Vries algebras, in which we do split the dual space of a de Vries algebra $(B, \prec)$ in two parts: the Stone dual of $B$ and the dual of $\prec$. If $X$ is the de Vries dual of $(B, \prec)$, then the Stone dual $Y$ of $B$ is the Gleason cover of $X$ [3]. We show that the irreducible map $\pi: Y \rightarrow X$ gives rise to what we call an irreducible equivalence relation $R$ on $Y$, which is the dual of $\prec$. It follows that compact Hausdorff spaces are in 1-1 correspondence with pairs $(Y, R)$, where $Y$ is an extremally disconnected compact Hausdorff space and $R$ is an irreducible equivalence relation on $Y$. We call such pairs Gleason spaces, and introduce the category of Gleason spaces, where morphisms are relations rather than functions, and composition is not relation composition. We prove that the category of Gleason spaces is equivalent to the category of compact Hausdorff spaces and continuous maps, and is dually equivalent to the category of de Vries algebras and de Vries morphisms, thus providing an alternate "modal-like" duality for de Vries algebras.

The paper is organized as follows. In Section 2, to set the scene, we recall modal algebras and quasi-modal algebras, as well as precontact relations, and introduce their dual concept of subordinations. Among many examples of subordinations, one of the key examples is that of de Vries' compingent relations. As follows from $[13,16]$ (see also [8, 17]), subordinations on a Boolean algebra $B$ correspond to closed relations on the Stone space of $B$. We extend this correspondence to a full categorical duality, which subsumes the duality of [13] (see Remark 2.23). In Section 3 we show that on objects the duality of Section 2 can be derived from the generalized Jónsson-Tarski duality. In Section 4 we prove that modally definable subordinations are dually described by means of Esakia relations. As a corollary, we derive the well-known duality between the categories of modal algebras and modal spaces. In Section 5 we show that a subordination is a lattice subordination iff its dual relation is a Priestley quasi-order. The duality result of [5] follows as a corollary. Finally, in Section 6 we introduce irreducible equivalence relations, Gleason spaces, and we give a "modal-like" alternative to de Vries duality. 


\section{Precontact, Subordination, and Quasi-modal Algebras}

One of the central concepts in the algebraic theory of modal logic is that of modal algebra. We recall (see, e.g., $[11,12,24]$ ) that a modal operator on a Boolean algebra $B$ is a unary function $\square: B \rightarrow B$ preserving finite meets (including 1), and that a modal algebra is a pair $(B, \square)$, where $B$ is a Boolean algebra and $\square$ is a modal operator on $B$. This concept was generalized in several directions.

Celani [13] generalized the concept of a modal operator to that of a quasi-modal operator. Let $\mathcal{I}(B)$ be the lattice of ideals of a Boolean algebra $B$.

Definition 2.1 ([13, Sec. 3]) A quasi-modal operator on $B$ is a function $\Delta: B \rightarrow \mathcal{I}(B)$ preserving finite meets, and a quasi-modal algebra is a pair $(B, \Delta)$, where $B$ is a Boolean algebra and $\Delta$ is a quasi-modal operator on $B$.

With a different (more geometric) motivation in mind, Düntsch and Vakarelov [18] (see also Dimov and Vakarelov [16]) introduced the concept of a precontact relation, generalizing that of a contact relation.

Definition 2.2 ([18, Sec. 3]) A proximity or a precontact relation on a Boolean algebra $B$ is a binary relation $\delta$ satisfying

(P1) $a \delta b \Rightarrow a, b \neq 0$.

(P2) $a \delta(b \vee c) \Leftrightarrow a \delta b$ or $a \delta c$.

(P3) $(a \vee b) \delta c \Leftrightarrow a \delta c$ or $b \delta c$.

The dual concept of a precontact relation is that of a subordination or a strong inclusion.

Definition 2.3 A subordination or a strong inclusion on a Boolean algebra $B$ is a binary relation $\prec$ satisfying

(S1) $0 \prec 0$ and $1 \prec 1$ (equivalently $0 \prec a \prec 1$ for each $a \in B$ );

(S2) $a \prec b, c$ implies $a \prec b \wedge c$;

(S3) $a, b \prec c$ implies $a \vee b \prec c$;

(S4) $a \leq b \prec c \leq d$ implies $a \prec d$.

Remark 2.4 As was pointed out to us by the referee, the name "subordination" was first introduced by Aleksandrov and Ponomarev [1] for the binary relations on the powerset of a set that are dual to Efremovic's proximities [26, Def. 1.7]. A pointfree version of this concept appeared in de Vries [14] under the name of a compingent relation (see Definition 2.11 below). Definition 2.3 generalizes this concept. We feel that it is convenient to keep the name subordination for this more general concept, and add additional adjectives when a stronger concept is introduced (see, for example, Definitions 2.10 and 2.11 below).

Remark 2.5 It is easy to see that precontact and subordination are dual concepts. Indeed, if $\delta$ is a precontact relation on $B$, then define $\prec_{\delta}$ by $a \prec_{\delta} b$ iff $a \not \supset b$. It is routine to check that $\prec_{\delta}$ is a subordination on $B$. Conversely, if $\prec$ is a subordination on $B$, then define $\delta_{\prec}$ by $a \delta_{\prec} b$ iff $a \nprec \neg b$. Again, it is routine to verify that $\delta_{\prec}$ is a precontact relation on $B$. Moreover, $a \delta b$ iff $a \delta_{\prec_{\delta}} b$, and $a \prec b$ iff $a \prec_{\delta_{\prec}} b$. Thus, precontact relations and subordinations on $B$ are in 1-1 correspondence. 
Remark 2.6 Precontact relations and subordinations are also in 1-1 correspondence with quasi-modal operators on $B$. Let $\prec$ be a subordination on $B$. For $S \subseteq B$, let

$$
\begin{aligned}
& \uparrow S=\{b \in B: \exists a \in S \text { with } a \prec b\} \\
& \Downarrow S=\{b \in B: \exists a \in S \text { with } b \prec a\} .
\end{aligned}
$$

Define $\Delta_{\prec}: B \rightarrow \mathcal{I}(B)$ by $\Delta_{\prec}(a)=\downarrow a$. It is easy to check that $\Delta_{\prec}$ is a quasi-modal operator on $B$. Conversely, if $\Delta$ is a quasi-modal operator on $B$, then define $\prec_{\Delta}$ by $a \prec_{\Delta} b$ iff $a \in \Delta(b)$. Again, it is easy to verify that $\prec_{\Delta}$ is a subordination on $B$. Moreover, $\Delta(a)=$ $\Delta_{\prec_{\Delta}}(a)$, and $a \prec b$ iff $a \prec_{\Delta_{\prec}} b$. Thus, subordinations and quasi-modal operators on $B$ are in 1-1 correspondence.

Instead of quasi-modal operators or precontact relations, we will mainly work with subordinations. Therefore, our ambient category will be that of Boolean algebras with subordinations.

Definition 2.7 Let Sub be the category whose objects are pairs $(B, \prec)$, where $B$ is a Boolean algebra and $\prec$ is a subordination on $B$, and whose morphisms are Boolean homomorphisms $h$ satisfying $a \prec b$ implies $h(a) \prec h(b)$.

Remark 2.8 It is easy to check that Sub is isomorphic to the category PCon whose objects are precontact algebras and whose morphisms are Boolean homomorphisms $h$ satisfying $h(a) \delta h(b)$ implies $a \delta b$. Another category isomorphic to Sub is the category qMA whose objects are quasi-modal algebras and whose morphisms are Boolean homomorphisms $h$ satisfying $h[\Delta a] \subseteq \Delta h(a)$.

We next show that modal operators give rise to special subordinations. Let $\square$ be a modal operator on a Boolean algebra $B$. Set $a \prec \square b$ provided $a \leq \square b$. Since $\square 1=1$, it is clear that $\prec \square$ satisfies (S1). As $\square(b \wedge c)=\square b \wedge \square c$, we also have that $\prec \square$ satisfies (S2). That $\prec \square$ satisfies (S3) is obvious, and since $\square$ is order-preserving, $\prec \square$ satisfies (S4). Therefore, $\prec \square$ is a subordination on $B$. Note that $\prec \square$ is a special subordination on $B$ that in addition satisfies the following condition: for each $a \in B$, the element $\square a$ is the largest element of the set $\{x \in B: x \prec \square a\}$.

Definition 2.9 We call a subordination $\prec$ on $B$ modally definable provided the set $\{x \in B$ : $x \prec a\}$ has a largest element for each $a \in B$.

We already saw that if $\square$ is a modal operator, then $\prec \square$ is a modally definable subordination. The converse is also true. If $\prec$ is a modally definable subordination on a Boolean algebra $B$, then define $\square_{\prec}: B \rightarrow B$ by

$$
\square_{\prec} a=\text { the largest element of }\{x \in B: x \prec a\} .
$$

By (S1), $\square_{\prec} 1=1$. In addition, by (S4), $\square_{\prec}(a \wedge b) \leq \square_{\prec} a \wedge \square_{\prec} b$, and by (S2) and (S4), $\square_{\prec} a \wedge \square_{\prec} b \leq \square_{\prec}(a \wedge b)$. Therefore, $\square_{\prec}$ is a modal operator on $B$. Moreover, $\square_{\prec} a=\square a$ and $a \prec \square_{\prec} b$ iff $a \prec b$. Thus, modal operators on $B$ are in 1-1 correspondence with modally definable subordinations on $B$.

Other examples of subordinations are the lattice subordinations of [5] and the compingent relations of [14]. 
Definition 2.10 ([5, Def. 2.1]) A subordination $\prec$ on a Boolean algebra $B$ is a lattice subordination if in addition $\prec$ satisfies

$$
a \prec b \text { implies that there exists } c \in B \text { with } c \prec c \text { and } a \leq c \leq b \text {. }
$$

Definition 2.11 ([14, Ch. 1]) A subordination $\prec$ on a Boolean algebra $B$ is a compingent relation or a de Vries subordination if in addition it satisfies:

(S5) $a \prec b$ implies $a \leq b$;

(S6) $\quad a \prec b$ implies $\neg b \prec \neg a$;

(S7) $a \prec b$ implies there is $c \in B$ with $a \prec c \prec b$;

(S8) $a \neq 0$ implies there is $b \neq 0$ with $b \prec a$.

\subsection{Duality}

By [13], there is a 1-1 correspondence between quasi-modal operators on a Boolean algebra $B$ and closed relations on the Stone space of $B$, and by [16], the same is true for precontact relations on $B .{ }^{1}$ From this it follows that the same characterization also holds for subordinations. A direct proof of this can be found in the first draft of this paper [8]. ${ }^{2}$ To keep the paper self-contained, we will briefly outline such a characterization below.

In $[16,17]$ it is shown that the $1-1$ correspondence between precontact relations on $B$ and closed relations on the Stone space of $B$ extends naturally to include appropriate isomorphisms, and in [13] it is shown that the 1-1 correspondence between quasi-modal operators and closed relations extends to a full duality of appropriate categories. For our purposes, a more general notion of morphism is required. In this section we will discuss how this results in a more general duality. In Remarks $2.14,2.17$, and 2.23 we compare our approach to that of [13] and [16, 17].

We call a binary relation $R$ on a topological space $X$ closed if $R$ is a closed set in the product topology on $X \times X$. As usual, for $U \subseteq X$, we use $R[U]$ and $R^{-1}[U]$ for the image and inverse image of $U$ with respect to $R$. A convenient characterization of closed quasi-orders (reflexive and transitive relations) on compact Hausdorff spaces is given in [10, Prop. 2.3]. This generalizes to the following characterization of arbitrary closed relations. (We skip the details and refer the interested reader to [8, Lem. 3.2].)

Lemma 2.12 Let $X$ be a compact Hausdorff space and let $R$ be a binary relation on $X$. The following conditions are equivalent.

(1) $R$ is a closed relation.

(2) For each closed subset $F$ of $X$, both $R[F]$ and $R^{-1}[F]$ are closed.

(3) If $A$ is an arbitrary subset of $X$, then $\overline{R[A]} \subseteq R[\bar{A}]$ and $\overline{R^{-1}[A]} \subseteq R^{-1}[\bar{A}]$.

(4) If $(x, y) \notin R$, then there is an open neighborhood $U$ of $x$ and an open neighborhood $V$ of $y$ such that $R[U] \cap V=\varnothing$.

\footnotetext{
${ }^{1}$ While the paper [16] contains no proofs, it was pointed out to us by the referee that the missing proofs were recently uploaded onto Math ArXiv [17].

${ }^{2}$ At the time, we were unaware of [13]. We would like to express our thanks to Ramon Jansana and Sergio Celani for bringing [13] to our attention.
} 
For $i=1,2$, let $R_{i}$ be a relation on $X_{i}$. Following [7], we call a map $f: X_{1} \rightarrow X_{2}$ stable provided $x R_{1} y$ implies $f(x) R_{2} f(y)$. It is easy to see that $f$ is stable iff $f\left(R_{1}[x]\right) \subseteq$ $R_{2}[f(x)]$ for each $x \in X_{1}$, which happens iff $R_{1}\left[f^{-1}(y)\right] \subseteq f^{-1}\left(R_{2}[y]\right)$ for each $y \in X_{2}$.

We recall that a subset $U$ of a topological space $X$ is clopen if it is both closed and open, and that $X$ is zero-dimensional if it has a basis of clopen sets. A Stone space is a compact, Hausdorff, zero-dimensional space. The celebrated Stone duality yields that the category of Boolean algebras and Boolean homomorphisms is dually equivalent to the category of Stone spaces and continuous maps.

Definition 2.13 Let StR be the category whose objects are pairs $(X, R)$, where $X$ is a Stone space and $R$ is a closed relation on $X$, and whose morphisms are continuous stable morphisms.

For a Boolean algebra $B$, let $X$ be the set of ultrafilters of $B$. For $a \in B$, set $\varphi(a)=\{x \in$ $X: a \in x\}$, and topologize $X$ by letting $\{\varphi(a): a \in B\}$ be a basis for the topology. The resulting space is called the Stone space of $B$ and is denoted $B_{*}$.

For $(B, \prec) \in \operatorname{Sub}$, let $(B, \prec)_{*}=(X, R)$, where $X$ is the Stone space of $B$ and $x R y$ iff $\uparrow x \subseteq y$. To see that $(X, R) \in \mathrm{StR}$, it is sufficient to show that $R$ is a closed relation on $X$. If $(x, y) \notin R$, then $\uparrow x \nsubseteq y$. Therefore, there are $a \in x$ and $b \notin y$ with $a \prec b$. But it is easy to check that $a \prec b$ implies $R[\varphi(a)] \subseteq \varphi(b)$. Set $U=\varphi(a)$ and $V=X-\varphi(b)$. Then $U$ is an open neighborhood of $x, V$ is an open neighborhood of $y$, and $R[U] \cap V=\varnothing$. Thus, by Lemma $2.12, R$ is a closed relation on $X$.

Remark 2.14 Let $(B, \prec) \in$ Sub and let $X$ be the Stone space of $B$. If $\Delta$ is the quasi-modal operator corresponding to $\prec$, then the relation $R$ on $X$ can alternatively be defined by $x R y$ iff $\{a \in B: \Delta(a) \cap x \neq \varnothing\} \subseteq y$ (see [13]). On the other hand, if $\delta$ is the precontact relation corresponding to $\prec$, then $R$ can be defined by $x R y$ iff $(\forall a \in x)(\forall b \in y)(a \delta b)$ (see [16]).

For $i=1,2$, let $\left(B_{i}, \prec_{i}\right) \in$ Sub and let $\left(X_{i}, R_{i}\right)=\left(B_{i}, \prec_{i}\right)_{*}$. For a morphism $h: B_{1} \rightarrow$ $B_{2}$ in Sub, let $h_{*}: X_{2} \rightarrow X_{1}$ be given by $h_{*}(x)=h^{-1}(x)$.

Lemma 2.15 If $h$ is a morphism in Sub, then $h_{*}$ is a morphism in StR.

Proof By Stone duality, $h_{*}$ is a well-defined continuous map. Suppose $x, y \in X_{2}$ with $x R_{2} y$. Then $\uparrow_{2} x \subseteq y$. Let $b \in \uparrow_{1} h^{-1}(x)$. So there is $a \in h^{-1}(x)$ with $a \prec_{1} b$. Since $h$ is a morphism in Sub, we have $h(a) \prec_{2} h(b)$. Therefore, $h(b) \in \uparrow_{2} x$. This implies $h(b) \in y$. Thus, $b \in h^{-1}(y)$, yielding ${ }^{{ }_{1}} h^{-1}(x) \subseteq h^{-1}(y)$. Consequently, $h_{*}$ is a stable continuous map, hence a morphism in StR.

Definition 2.16 Define $(-)_{*}$ : Sub $\rightarrow$ StR as follows. If $(B, \prec) \in$ Sub, then $(B, \prec)_{*}=$ $(X, R)$, and if $h$ is a morphism in Sub, then $h_{*}=h^{-1}$. It is straightforward to see that $(-)_{*}$ is a well-defined contravariant functor.

For a topological space $X$, let $\operatorname{Clop}(X)$ be the set of clopen subsets of $X$. Then it is well known and easy to see that $\operatorname{Clop}(X)$ is a Boolean algebra with respect to the set-theoretic operations of union, intersection, and complement.

For $(X, R) \in \operatorname{StR}$, let $(X, R)^{*}=(\operatorname{Clop}(X), \prec)$, where $U \prec V$ iff $R[U] \subseteq V$. It is straightforward to check that $\prec$ is a subordination on $\operatorname{Clop}(X)$. Therefore, $(X, R)^{*} \in \mathrm{Sub}$. 
Remark 2.17 If $(X, R) \in \mathrm{StR}$, then the quasi-modal operator $\Delta$ on $\operatorname{Clop}(X)$ corresponding to $\prec$ is defined by $\Delta(V)=\{U \in \operatorname{Clop}(X): R[U] \subseteq V\}$ (see [13]). On the other hand, the precontact relation $\delta$ corresponding to $\prec$ is defined by $U \delta V$ iff $R[U] \cap V \neq \varnothing$ (see [16]).

For $i=1,2$, let $\left(X_{i}, R_{i}\right) \in \mathrm{StR}$ and let $\left(B_{i}, \prec_{i}\right)=\left(X_{i}, R_{i}\right)^{*}$. For a morphism $f: X_{1} \rightarrow$ $X_{2}$ in StR, let $f^{*}: \operatorname{Clop}\left(X_{2}\right) \rightarrow \operatorname{Clop}\left(X_{1}\right)$ be given by $f^{*}(U)=f^{-1}(U)$.

Lemma 2.18 If $f$ is a morphism in StR, then $f^{*}$ is a morphism in Sub.

Proof It follows from Stone duality that $f^{*}$ is a Boolean homomorphism. Let $U, V \in$ $\operatorname{Clop}\left(X_{2}\right)$ with $U \prec_{2} V$. Then $R_{2}[U] \subseteq V$. This implies $f^{-1}\left(R_{2}[U]\right) \subseteq f^{-1}(V)$. Since $f$ is a stable map, $R_{1}\left[f^{-1}(U)\right] \subseteq f^{-1}\left(R_{2}[U]\right)$. Therefore, $R_{1}\left[f^{-1}(U)\right] \subseteq f^{-1}(V)$. Thus, $f^{-1}(U) \prec f^{-1}(V)$, and hence $f^{*}$ is a morphism in Sub.

Definition 2.19 Define $(-)^{*}: \operatorname{StR} \rightarrow$ Sub as follows. If $(X, R) \in \mathrm{StR}$, then $(X, R)^{*}=$ $(\operatorname{Clop}(X), \prec)$, and if $f$ is a morphism in StR, then $f^{*}=f^{-1}$. It is straightforward to see that $(-)^{*}$ is a contravariant functor.

Lemma 2.20 Let $(B, \prec) \in$ Sub and let $\varphi: B \rightarrow\left(B_{*}\right)^{*}$ be the Stone map. Then $a \prec b$ iff $\varphi(a) \prec \varphi(b)$.

Proof Let $a, b \in B$. If $a \prec b$, then $R[\varphi(a)] \subseteq \varphi(b)$, so $\varphi(a) \prec \varphi(b)$. If $a \nprec b$, then $b \notin \uparrow a$. Since $\prec$ is a subordination, it is easy to see that $\uparrow a$ is a filter. Therefore, by the ultrafilter theorem, there is an ultrafilter $x$ such that $\uparrow a \subseteq x$ and $b \notin x$. By Zorn's lemma, there is an ultrafilter $y$ such that $a \in y$ and $\uparrow y \subseteq x$ (see [8, Claim to Lem. 3.14]). Thus, there is $y \in B_{*}$ such that $y \in \varphi(a)$ and $y R x$. This gives $x \in R[\varphi(a)]$. On the other hand, $x \notin \varphi(b)$. Consequently, $R[\varphi(a)] \nsubseteq \varphi(b)$, yielding $\varphi(a) \nprec \varphi(b)$.

For a Stone space $X$, define $\psi: X \rightarrow\left(X^{*}\right)_{*}$ by $\psi(x)=\{U \in \operatorname{Clop}(X): x \in U\}$. It follows from Stone duality that $\psi$ is a homeomorphism.

Lemma 2.21 Let $(X, R) \in \mathrm{StR}$ and let $\psi: X \rightarrow\left(X^{*}\right)_{*}$ be given as above. Then $x$ Ry iff $\psi(x) R \psi(y)$.

Proof First suppose that $x R y$. To see that $\psi(x) R \psi(y)$ we must show that $\uparrow \psi(x) \subseteq \psi(y)$. Let $V \in \uparrow \psi(x)$. Then there is $U \in \psi(x)$ with $U \prec V$. Therefore, $x \in U$ and $R[U] \subseteq V$. Thus, $y \in V$, so $\uparrow \psi(x) \subseteq \psi(y)$, and hence $\psi(x) R \psi(y)$.

Conversely, suppose that $(x, y) \notin R$. Since $X$ has a basis of clopens and $R$ is a closed relation, by Lemma 2.12, there exist a clopen neighborhood $U$ of $x$ and a clopen neighborhood $W$ of $y$ such that $R[U] \cap W=\varnothing$. Set $V=X-W$. Then $U \in \psi(x)$, $V \notin \psi(y)$, and $R[U] \subseteq V$. Therefore, $U \prec V$, so $V \in{ }^{\uparrow} \psi(x)$, but $V \notin \psi(y)$. Thus, $(\psi(x), \psi(y)) \notin R$.

Theorem 2.22 The categories Sub and StR are dually equivalent.

Proof By Definition 2.16, $(-)_{*}$ : Sub $\rightarrow$ StR is a well-defined contravariant functor, and by Definition $2.19,(-)^{*}: \mathrm{StR} \rightarrow$ Sub is a well-defined contravariant functor. By Stone duality and Lemmas 2.20 and 2.21 , each $(B, \prec) \in \operatorname{Sub}$ is isomorphic in Sub to $\left((B, \prec)_{*}\right)^{*}$ 
and each $(X, R) \in \mathrm{StR}$ is isomorphic in StR to $\left((X, R)^{*}\right)_{*}$. That these isomorphisms are natural is easy to see. Thus, Sub is dually equivalent to StR.

Remark 2.23 Since Sub is isomorphic to qMA and PCon, it follows that each of these categories is dually equivalent to StR. In particular, each quasi-modal algebra $(B, \Delta)$ is represented as $(\operatorname{Clop}(X), \Delta)[13$, Thm. 9], and each precontact algebra $(B, \delta)$ is represented as $(\operatorname{Clop}(X), \delta)[16$, Thm. 3].

In [13], following the tradition in modal logic, Celani works with a stronger concept of morphisms between quasi-modal algebras, which satisfy that $\Delta h(a)$ is the ideal generated by $h[\Delta a]$. Let qMA ${ }^{c}$ be the (non-full) subcategory of qMA having the same objects as qMA but whose morphisms satisfy that $h[\Delta a]$ is generated by $\Delta h(a)$. Also, let StR ${ }^{\mathrm{b}}$ be the (non-full) subcategory of StR having the same objects as StR but whose morphisms are in addition bounded morphisms (that is, $f\left(R_{1}[x]\right)=R_{2}[f(x)]$ for each $x \in X_{1}$ ). Then the dual equivalence of qMA and StR restricts to the dual equivalence of qMA ${ }^{c}$ and $S R^{b}$ of [13].

In $[16,17]$ the $1-1$ correspondence between objects of PCon and StR is extended naturally to include isomorphisms in Con and StR. This correspondence further extends to a dual equivalence of PCon and StR. While PCon is isomorphic to Sub, it appears that the definition of morphisms is more intuitive when working with subordinations since they are required to preserve subordinations. On the other hand, when working with precontact relations, we have to require that morphisms reflect precontact relations (see Remark 2.8).

\section{Subordinations, Strict Implications, and Jónsson-Tarski Duality}

In this section we show that on objects the duality of the previous section can also be derived from the generalized Jónsson-Tarski duality.

Definition 3.1 Let $B$ be a Boolean algebra and let $\mathbf{2}$ be the two element Boolean algebra. We call a map $\rightarrow: B \times B \rightarrow \mathbf{2}$ a strict implication if it satisfies

(I1) $0 \rightarrow a=a \rightarrow 1=1$.

(I2) $(a \vee b) \rightarrow c=(a \rightarrow c) \wedge(b \rightarrow c)$.

(I3) $a \rightarrow(b \wedge c)=(a \rightarrow b) \wedge(a \rightarrow c)$.

Example 3.2 Let $\prec$ be a subordination on a Boolean algebra $B$. Define $\rightarrow_{\prec}: B \times B \rightarrow \mathbf{2}$ by

$$
a \rightarrow_{\prec} b= \begin{cases}1 & \text { if } a \prec b, \\ 0 & \text { otherwise }\end{cases}
$$

It is easy to see that $\rightarrow_{\prec}$ is a strict implication. Conversely, if $\rightarrow: B \times B \rightarrow \mathbf{2}$ is a strict implication, then define $\prec \rightarrow \subseteq B \times B$ by

$$
a \prec \rightarrow b \text { iff } a \rightarrow b=1 \text {. }
$$

It is straightforward to see that $\prec \rightarrow$ is a subordination on $B$. Moreover, $a \prec b$ iff $a \prec \rightarrow_{<} b$ and $a \rightarrow b=a \rightarrow \prec \rightarrow b$. Thus, there is a 1-1 correspondence between subordinations and strict implications on $B$. 
This observation opens the door for obtaining the duality for subordinations from Jónsson-Tarski duality [23]. Let $A, B, C$ be Boolean algebras and $X, Y, Z$ be their respective Stone spaces. Suppose that $f: A \times B \rightarrow C$ is a map. Following the terminology of [28], we call $f$ a meet-hemiantimorphism in the first coordinate provided

- $f(0, b)=1$,

- $f(a \vee b, c)=f(a, c) \wedge f(b, c)$;

and a meet-hemimorphism in the second coordinate provided

- $f(a, 1)=1$,

- $f(a, b \wedge c)=f(a, c) \wedge f(b, c)$.

By the generalized Jónsson-Tarski duality [21, 28], such maps are dually described by special ternary relations $S \subseteq X \times Y \times Z$. For $z \in Z$, let

$$
S^{-1}[z]:=\{(x, y) \in X \times Y:(x, y, z) \in S\},
$$

and for $U \in \operatorname{Clop}(X)$ and $V \in \operatorname{Clop}(Y)$, let

$$
\square_{S}(U, V):=\{z \in Z:(\forall x \in X)(\forall y \in Y)[(x, y, z) \in S \Rightarrow x \notin U \text { or } y \in V]\} .
$$

Definition 3.3 We call $S \subseteq X \times Y \times Z$ a JT-relation (Jónsson-Tarski relation) provided

(JT1) $S^{-1}[z]$ is closed for each $z \in Z$,

(JT2) $\square_{S}(U, V)$ is clopen for each $U \in \operatorname{Clop}(X)$ and $V \in \operatorname{Clop}(Y)$.

By the generalized Jónsson-Tarski duality [21, 28], the dual ternary relation $S \subseteq X \times$ $Y \times Z$ of $f: A \times B \rightarrow C$ is given by

$$
(x, y, z) \in S \text { iff }(\forall a \in A)(\forall b \in B)(f(a, b) \in z \text { implies } a \notin x \text { or } b \in y) ;
$$

and the dual map $f: \operatorname{Clop}(X) \times \operatorname{Clop}(Y) \rightarrow \operatorname{Clop}(Z)$ of $S \subseteq X \times Y \times Z$ is given by

$$
f(U, V)=\square_{S}(U, V) .
$$

Now let $\rightarrow$ be a strict implication on a Boolean algebra $B$. By Definition $3.1, \rightarrow$ is a meet-hemiantimorphism in the first coordinate and a meet-hemimorphism in the second coordinate. Let $X$ be the Stone space of $B$. The Stone space of $\mathbf{2}$ is the singleton discrete space $\{z\}$, where $z=\{1\}$ is the only ultrafilter of $\mathbf{2}$. Therefore, the dual ternary relation $S \subseteq X \times X \times\{z\}$ of $\rightarrow$ is given by

$$
(x, y, z) \in S \text { iff }(\forall a, b \in B)(a \rightarrow b=1 \text { implies } a \notin x \text { or } b \in y) .
$$

The ternary relation $S$ gives rise to the binary relation $R \subseteq X \times X$ by setting

$$
x R y \text { iff }(x, y, 1) \in S .
$$

If $\prec$ is the subordination corresponding to the strict implication $\rightarrow$, then $a \prec b$ iff $a \rightarrow b=$ 1. Therefore, the binary relation $R$ is given by

$$
x R y \text { iff }(\forall a, b \in B)(a \prec b \text { implies } a \notin x \text { or } b \in y) .
$$

Proposition 3.4 Let $\prec$ be a subordination on a Boolean algebra $B$, and let $(X, R)$ be the dual of $(B, \prec)$. Then $\uparrow x \subseteq y$ iff $(\forall a, b \in B)(a \prec b$ implies $a \notin x$ or $b \in y)$. 
Proof First suppose that $\uparrow x \subseteq y$. Let $a \prec b$ and $a \in x$. Then $b \in \uparrow x$, so $b \in y$. Conversely, suppose $(\forall a, b \in B)(a \prec b$ implies $a \notin x$ or $b \in y)$. If $b \in \uparrow x$, then there is $a \in x$ with $a \prec b$. Therefore, $y \in b$, and hence $\uparrow x \subseteq y$.

Applying Proposition 3.4 then yields

$$
x R y \text { iff } \uparrow x \subseteq y .
$$

Consequently, the dual binary relation $R$ of a subordination $\prec$ can be described from the dual ternary relation $S$ of the corresponding strict implication. In fact, if $S \subseteq X \times X \times\{z\}$ is a JT-relation, then (JT2) is redundant, while (JT1) means that $R$ is a closed relation.

The converse is also true. Given a closed relation $R$ on a Stone space $X$, define the ternary relation $S \subseteq X \times X \times\{z\}$ by

$$
(x, y, z) \in S \text { iff } x R y .
$$

Since $R$ is a closed relation, $S$ satisfies (JT1), and $S$ satisfies (JT2) trivially, hence $S$ is a JT-relation. Let $\rightarrow: \operatorname{Clop}(X) \times \operatorname{Clop}(X) \rightarrow \mathbf{2}$ be the corresponding strict implication. Then

$$
U \rightarrow V= \begin{cases}1 & \text { if }(\forall x, y \in X)(x R y \Rightarrow x \notin U \text { or } y \in V) \\ 0 & \text { otherwise. }\end{cases}
$$

Proposition 3.5 Let $X$ be a Stone space, $R$ be a closed relation on $X$, and $U, V \in \operatorname{Clop}(X)$. Then $R[U] \subseteq V$ iff $(\forall x, y \in X)(x R y$ implies $x \notin U$ or $y \in V)$.

Proof First suppose that $R[U] \subseteq V, x R y$, and $x \in U$. Then $y \in R[U]$, so $y \in V$. Conversely, suppose that $(\forall x, y \in X)(x R y$ implies $x \notin U$ or $y \in V)$. If $y \in R[U]$, then there is $x \in U$ with $x R y$. Therefore, $y \in V$, and hence $R[U] \subseteq V$.

If $\prec$ is the subordination corresponding to $\rightarrow$, then it follows from Proposition 3.5 that $U \prec V$ iff $R[U] \subseteq V$ iff $U \rightarrow V=1$. This shows that on objects our duality for subordinations is equivalent to a special case of the generalized Jónsson-Tarski duality.

Remark 3.6 A homomorphism between two Boolean algebras with strict implication $\left(B_{1}, \rightarrow_{1}\right)$ and $\left(B_{2}, \rightarrow_{2}\right)$ is a Boolean homomorphism $h: B_{1} \rightarrow B_{2}$ such that $h\left(a \rightarrow_{1}\right.$ $b)=h(a) \rightarrow_{2} h(b)$. On the other hand, a morphism between two Boolean algebras with subordination $\left(B_{1}, \prec_{1}\right)$ and $\left(B_{2}, \prec_{2}\right)$ is a Boolean homomorphism $h: B_{1} \rightarrow B_{2}$ such that $a \prec_{1} b \Rightarrow h(a) \prec_{2} h(b)$. It is easy to verify that $a \prec_{1} b \Rightarrow h(a) \prec_{2} h(b)$ is equivalent to $h\left(a \rightarrow_{1} b\right) \leq h(a) \rightarrow_{2} h(b)$, while $h\left(a \rightarrow_{1} b\right)=h(a) \rightarrow_{2} h(b)$ is equivalent to $a \prec_{1} b$ iff $h(a) \prec_{2} h(b)$. Thus, continuous stable morphisms dually correspond to $h\left(a \rightarrow_{1} b\right) \leq h(a) \rightarrow_{2} h(b)$, while the equality $h\left(a \rightarrow_{1} b\right)=h(a) \rightarrow_{2} h(b)$ requires an additional condition: If $y, u \in X_{2}$ and $y R_{2} u$, then there exist $x, z \in X_{1}$ such that $x R_{1} z$, $f(x)=y$, and $f(z)=u$. This is equivalent to $f: X_{1} \rightarrow X_{2}$ being a bounded morphism with respect to the ternary relations $S_{1}$ on $X_{1}$ and $S_{2}$ on $X_{2}$.

\section{Modally Definable Subordinations and Esakia Relations}

In this section we show that modally definable subordinations dually correspond to Esakia relations, and derive the well-known duality between the categories of modal algebras and modal spaces from the duality of Section 2. 
Definition 4.1 Let $X$ be a Stone space. We call a binary relation $R$ on $X$ an Esakia relation provided $R[x]$ is closed for each $x \in X$ and $U \in \operatorname{Clop}(X)$ implies $R^{-1}[U] \in \operatorname{Clop}(X)$.

\section{Remark 4.2}

(1) Let $\mathcal{V}(X)$ be the Vietoris space of $X$. It is well known (see, e.g., [19]) that $R$ is an Esakia relation iff the map $\rho_{R}: X \rightarrow \mathcal{V}(X)$ given by $\rho(x)=R[x]$ is a well-defined continuous map. Because of this, Esakia relations are also called continuous relations.

(2) It is easy to see that Esakia relations are exactly the inverses of binary JT-relations with the same source and target (see, e.g., [21]). Inverses of binary JT-relations with not necessarily the same source and target were first studied by Halmos [22].

It is a standard argument that each Esakia relation is closed, but there exist closed relations that are not Esakia relations. In fact, for a closed relation $R$ on a Stone space $X$, the following are equivalent:

(1) $R$ is an Esakia relation.

(2) $U \in \operatorname{Clop}(X)$ implies $R^{-1}[U] \in \operatorname{Clop}(X)$.

(3) $U$ open implies $R^{-1}[U]$ is open.

Therefore, Esakia relations are special closed relations. We show that they dually correspond to modally definable subordinations. Our proof is a generalization of [5, Lem. 5.6].

\section{Lemma 4.3}

(1) Suppose that $(B, \prec) \in \operatorname{Sub}$ and $(X, R)=(B, \prec)_{*}$. If $\prec$ is modally definable, then $R$ is an Esakia relation.

(2) Suppose that $R$ is an Esakia relation on a Stone space $X$ and $(B, \prec)=(X, R)^{*}$. Then $\prec$ is modally definable.

Proof (1) Suppose that $\prec$ is modally definable and $\square_{\prec}$ is the largest element of $\{b \in B$ : $b \prec a\}$.

Claim $\varphi\left(\square_{\prec} a\right)=X-R^{-1}[X-\varphi(a)]$.

Proof of Claim We have $x \in X-R^{-1}[X-\varphi(a)]$ iff $R[x] \subseteq \varphi(a)$. This is equivalent to $(\forall y \in X)(\uparrow x \subseteq y \Rightarrow a \in y)$. Since $\uparrow x$ is a filter, by the ultrafilter theorem, it is the intersection of the ultrafilters containing it. Therefore, the last condition is equivalent to $a \in \uparrow_{x}$. Because $\square_{\prec} a$ is the largest element of $\{b \in B: b \prec a\}$, this is equivalent to $\square_{\prec} a \in x$, which means that $x \in \varphi\left(\square_{\prec} a\right)$. Thus, $\varphi\left(\square_{\prec} a\right)=X-R^{-1}[X-\varphi(a)]$.

Now, let $U \in \operatorname{Clop}(X)$. Then $X-U \in \operatorname{Clop}(X)$, so there is $a \in B$ with $\varphi(a)=X-U$. Therefore, $\varphi\left(\square_{\prec} a\right)=X-R^{-1}[X-\varphi(a)]=X-R^{-1}[U]$. This yields $X-R^{-1}[U] \in$ Clop $(X)$, so $R^{-1}[U] \in \operatorname{Clop}(X)$. Since $R$ is also a closed relation, we conclude that $R$ is an Esakia relation.

(2) Let $U \in \operatorname{Clop}(X)$. We show that $X-R^{-1}[X-U]$ is the largest element of $\{V \in$ $\operatorname{Clop}(X): V \prec U\}$. Let $y \in R\left[X-R^{-1}[X-U]\right]$. Then there is $x \in X-R^{-1}[X-U]$ with $x R y$. From $x \in X-R^{-1}[X-U]$ it follows that $R[x] \subseteq U$. Therefore, $y \in U$, yielding $X-R^{-1}[X-U] \prec U$. Suppose that $V \in \operatorname{Clop}(X)$ with $V \prec U$. Then $R[V] \subseteq U$, so $V \subseteq X-R^{-1}[X-U]$. Thus, $X-R^{-1}[X-U]$ is the largest element of $\{V \in \operatorname{Clop}(X)$ : $V \prec U\}$, and hence $\prec$ is modally definable. 
We recall that a modal space is a pair $(X, R)$, where $X$ is a Stone space and $R$ is an Esakia relation on $X$. Modal spaces are also known as descriptive frames. They are fundamental objects in the study of modal logic as they serve as dual spaces of modal algebras (see, e.g., $[11,12,24])$.

Let $\mathrm{MS}^{\text {st }}$ be the category whose objects are modal spaces and whose morphisms are continuous stable morphisms. Let also MSub be the full subcategory of Sub consisting of the objects $(B, \prec)$ of Sub in which $\prec$ is modally definable. It is an immediate consequence of Theorem 2.22 and Lemma 4.3 that MSub is dually equivalent to MS $^{\text {st }}$.

But modal logicians are more interested in bounded morphisms rather than stable morphisms since they dually correspond to modal algebra homomorphisms. We recall that a modal homomorphism is a Boolean homomorphism $h: B_{1} \rightarrow B_{2}$ such that $h\left(\square_{1} a\right)=$ $\square_{2} h(a)$. We also recall that a bounded morphism is a stable morphism $f: X_{1} \rightarrow X_{2}$ such that $f(x) R_{2} y$ implies the existence of $z \in X_{1}$ with $x R_{1} z$ and $f(z)=y$ (equivalently $f\left(R_{1}[x]\right)=R_{2}[f(x)]$ for each $\left.x \in X_{1}\right)$. Let MA be the category whose objects are modal algebras and whose morphisms are modal homomorphisms, and let MS be the category whose objects are modal spaces and whose morphisms are continuous bounded morphisms. (Note that MS is not a full subcategory of $\mathrm{MS}^{\text {st }}$.) It is a standard result in modal logic that MA is dually equivalent to MS. We next show how to obtain this dual equivalence from our results.

Let $h: B_{1} \rightarrow B_{2}$ be a morphism in MSub. For $a \in B_{1}$, let $\square_{1} a$ be the largest element of $\left\{x \in B_{1}: x \prec_{1} a\right\}$, and for $b \in B_{2}$, let $\square_{2} b$ be the largest element of $\left\{y \in B_{2}: y \prec_{2} b\right\}$. Since $\square_{1} a \prec_{1} a$, we have $h\left(\square_{1} a\right) \prec_{2} h(a)$. Therefore, $h\left(\square_{1} a\right) \leq_{2} \square_{2} h(a)$. Conversely, suppose that $h$ is a Boolean homomorphism satisfying $h\left(\square_{1} a\right) \leq_{2} \square_{2} h(a)$ for each $a \in B_{1}$. Let $a, b \in B_{1}$ with $a \prec_{1} b$. Then $a \leq_{1} \square_{1} b$. Therefore, $h(a) \leq_{2} h\left(\square_{1} b\right) \leq_{2} \square_{2} h(b)$. Thus, $h(a) \prec_{2} h(b)$, and $h$ is a morphism in MSub.

We call a morphism $h$ in MSub a modal homomorphism if $h\left(\square_{1} a\right)=\square_{2} h(a)$. Let MSub ${ }^{\mathrm{m}}$ be the category whose objects are the objects of MSub and whose morphisms are modal homomorphisms. Then MSub ${ }^{m}$ is a non-full subcategory of MSub, and it is evident that $\mathrm{MSub}^{\mathrm{m}}$ is isomorphic to MA.

We show that $\mathrm{MSub}^{\mathrm{m}}$ is dually equivalent to MS. For this, taking into account the dual equivalence of MSub and $\mathrm{MS}^{\mathrm{st}}$, it is sufficient to see that if $h$ is a morphism in $\mathrm{MSub}^{\mathrm{m}}$, then $h_{*}$ is a morphism in MS, and that if $f$ is a morphism in MS, then $f^{*}$ is a morphism in MSub $^{m}$. This is proved in the next lemma, which generalizes [5, Lem. 5.7].

\section{Lemma 4.4}

(1) Let $\left(B_{1}, \prec_{1}\right),\left(B_{2}, \prec_{2}\right) \in \mathrm{MSub}^{\mathrm{m}}$ and $h: B_{1} \rightarrow B_{2}$ be a morphism in $\mathrm{MSub}^{\mathrm{m}}$. Then $h_{*}$ is a morphism in MS.

(2) Let $\left(X_{1}, R_{1}\right),\left(X_{2}, R_{2}\right) \in \mathrm{MS}$ and $f: X_{1} \rightarrow X_{2}$ be a morphism in MS. Then $f^{*}$ is a morphism in $\mathrm{MSub}^{\mathrm{m}}$.

Proof (1) From the dual equivalence of MSub and $\mathrm{MS}^{\text {st }}$ we know that $h_{*}$ is continuous and stable. Suppose that $h_{*}(x) R_{1} y$. Then ${ }^{\uparrow_{1}} h^{-1}(x) \subseteq y$. Let $F$ be the filter generated by $\uparrow_{2} x \cup h(y)$ and let $I$ be the ideal generated by $h\left(B_{1}-y\right)$. If $F \cap I \neq \varnothing$, then there exist $a \in \uparrow_{2} x, b \in y$, and $c \notin y$ such that $a \wedge_{2} h(b) \leq_{2} h(c)$. Therefore, $a \leq_{2} h\left(b \rightarrow_{1} c\right)$. From $a \in \uparrow_{2} x$ it follows that there is $d \in x$ with $d \prec_{2} a$. So $d \leq_{2} \square_{2} a$. But $a \leq_{2} h\left(b \rightarrow_{1} c\right)$ implies $\square_{2} a \leq_{2} \square_{2} h\left(b \rightarrow_{1} c\right)=h\left(\square_{1}\left(b \rightarrow_{1} c\right)\right)$. This yields $\square_{1}\left(b \rightarrow_{1} c\right) \in h^{-1}(x)$, so $b \rightarrow{ }_{1} c \in{ }^{{ }_{1}} h^{-1}(x) \subseteq y$, which is a contradiction since $b \in y$ and $c \notin y$. Thus, $F \cap I=\varnothing$, and by the ultrafilter theorem, there is an ultrafilter $z$ containing $F$ and missing $I$. From 
$\uparrow_{2} x \subseteq z$ it follows that $x R_{2} z$, and from $h(y) \subseteq z$ and $h\left(B_{1}-y\right) \cap z=\varnothing$ it follows that $h^{-1}(z)=y$. Consequently, there is $z$ such that $x R_{2} z$ and $h_{*}(z)=y$, yielding that $h_{*}$ is a morphism in MS.

(2) From the dual equivalence of MSub and $\mathrm{MS}^{\text {st }}$ we know that $f^{*}$ is a Boolean homomorphism satisfying $U \prec_{2} V$ implies $f^{*}(U) \prec_{1} f^{*}(V)$ for each $U, V \in \operatorname{Clop}\left(X_{2}\right)$. Therefore, $f^{*}\left(\square_{2} U\right) \leq_{1} \square_{1} f^{*}(U)$ for each $U \in \operatorname{Clop}\left(X_{2}\right)$. Suppose that $x \in \square_{1} f^{*}(U)$. Then $R_{1}[x] \subseteq f^{-1}(U)$, so $f\left(R_{1}[x]\right) \subseteq U$. Since $f$ is a bounded morphism, $f\left(R_{1}[x]\right)=$ $R_{2}[f(x)]$. Therefore, $R_{2}[f(x)] \subseteq U$, yielding $f(x) \in \square_{2} U$. Thus, $x \in f^{-1}\left(\square_{2} U\right)$. This implies that $f^{*}\left(\square_{2} U\right)=\square_{1} f^{*}(U)$ for each $U \in \operatorname{Clop}\left(X_{2}\right)$, hence $f^{*}$ is a morphism in MSub $^{\mathrm{m}}$.

As a consequence, we obtain that $\mathrm{MSub}^{\mathrm{m}}$ is dually equivalent to MS, and since $\mathrm{MSub}^{\mathrm{m}}$ is isomorphic to MA, as a corollary, we obtain the well-known dual equivalence of MA and MS. To summarize:

\section{Theorem 4.5}

(1) MSub is dually equivalent to $\mathrm{MS}^{\text {st }}$.

(2) $\mathrm{MSub}^{\mathrm{m}}$ is isomorphic to MA.

(3) $\mathrm{MSub}^{\mathrm{m}}$ is dually equivalent to MS, hence MA is dually equivalent to MS.

In modal logic, modal algebras corresponding to reflexive, transitive, and/or symmetric modal spaces play an important role. In [13], quasi-modal algebras $(B, \Delta)$ were characterized such that the dual closed relation $R$ on the Stone space $X$ of $B$ is reflexive, transitive, and/or symmetric. The same characterization was given in [18] (see also [16]) in terms of precontact relations on $B$. Namely, consider the following axioms:

(P4) $a \neq 0$ implies $a \delta a$.

(P5) $a \delta b$ implies $b \delta a$.

(P6) $a \not \delta b$ implies there is $c \in B$ with $a \not \delta c$ and $\neg c \not \delta b$.

Let $(B, \delta)$ be a precontact algebra and $(X, R)$ be its dual. Then $R$ is reflexive iff $(B, \delta)$ satisfies (P4), $R$ is symmetric iff $(B, \delta)$ satisfies (P5), and $R$ is transitive iff $(B, \delta)$ satisfies (P6).

It is easy to see that if $\prec$ is the subordination corresponding to the precontact relation $\delta$, then (S5) corresponds to (P4), (S6) corresponds to (P5), and (S7) corresponds to (P6). This immediately yields the following lemma.

Lemma 4.6 (cf. $[13,18])$ Let $(B, \prec) \in \operatorname{Sub}$ and let $(X, R)$ be the dual of $(B, \prec)$.

(1) $R$ is reflexive iff $\prec$ satisfies ( $S 5)$.

(2) $R$ is symmetric iff $\prec$ satisfies (S6).

(3) $R$ is transitive iff $\prec$ satisfies (S7).

Remark 4.7 For a direct proof of Lemma 4.6, without first switching from $\prec$ to $\delta$ or $\Delta$, consult [8, Lem. 6.1]

Remark 4.8 Axioms (S5), (S6), and (S7) correspond to elementary conditions on $R$. Developing a general theory which characterizes the class of axioms for subordinations corresponding to elementary conditions on $R$ is closely related to the field of Sahlqvist theory in modal logic $[11,12]$. In fact, by the perspective of Section 3, Lemma 4.6 can be seen as an instance of the standard Sahlqvist theory, applied to a binary modality. A Sahlqvist correspondence for logics corresponding to precontact algebras is developed in [2]. 


\section{Definition 4.9}

(1) Let SubK4 be the full subcategory of Sub consisting of the $(B, \prec) \in$ Sub that satisfy (S7).

(2) Let SubS4 be the full subcategory of Sub consisting of the $(B, \prec) \in$ Sub that satisfy (S5) and (S7).

(3) Let SubS5 be the full subcategory of Sub consisting of the $(B, \prec) \in$ Sub that satisfy (S5), (S6), and (S7).

Clearly SubS5 is a full subcategory of SubS4, and SubS4 is a full subcategory of SubK4.

\section{Definition 4.10}

(1) Let $\mathrm{StR}^{\mathrm{tr}}$ be the full subcategory of StR consisting of the $(X, R) \in \mathrm{StR}$, where $R$ is transitive.

(2) Let $\mathrm{StR}^{\mathrm{qo}}$ be the full subcategory of StR consisting of the $(X, R) \in \mathrm{StR}$, where $R$ is a quasi-order (that is, $R$ is reflexive and transitive).

(3) Let $\mathrm{StR}^{\mathrm{eq}}$ be the full subcategory of StR consisting of the $(X, R) \in \mathrm{StR}$, where $R$ is an equivalence relation.

Clearly $\operatorname{StR}^{\mathrm{eq}}$ is a full subcategory of $\operatorname{StR}^{\mathrm{qo}}$, and $\operatorname{StR}^{\mathrm{qo}}$ is a full subcategory of $\mathrm{StR}^{\mathrm{tr}}$. The next theorem is an immediate consequence of Theorem 2.22 and Lemma 4.3.

\section{Theorem 4.11}

(1) SubK4 is dually equivalent to $\mathrm{StR}^{\mathrm{tr}}$.

(2) SubS4 is dually equivalent to $\mathrm{StR}^{\text {qo }}$.

(3) SubS5 is dually equivalent to $\mathrm{StR}^{\mathrm{eq}}$.

Remark 4.12 We recall (see, e.g., $[16,18])$ that a precontact algebra $(B, \delta)$ is a contact algebra if it satisfies the following two axioms:

(P4) $a \neq 0$ implies $a \delta a$.

(P5) $a \delta b$ implies $b \delta a$.

Let Con be the full subcategory of PCon consisting of contact algebras. Since (P4) is the $\delta$-analogue of (S5) and (P5) is the $\delta$-analogue of (S6), Con is isomorphic to the full subcategory of Sub whose objects satisfy (S5) and (S6). By Lemma 4.6, Con is dually equivalent to the full subcategory of StR consisting of such $(X, R) \in \mathrm{StR}$, where $R$ is reflexive and symmetric.

Remark 4.13 We recall that a modal algebra $(B, \square)$ is a $\mathbf{K 4}$-algebra if $\square a \leq \square \square a$ for each $a \in B$; a K4-algebra is an S4-algebra if $\square a \leq a$ for each $a \in B$; and an S4-algebra is an S5-algebra if $a \leq \square \diamond a$ for each $a \in B$ (where, as usual, $\diamond a=\neg \square \neg a$ ). Let K4, S4, and S5 denote the categories of K4-algebras, S4-algebras, and S5-algebras, respectively.

We also let TRS be the category of transitive modal spaces, QOS be the category of quasiordered modal spaces, and EQS be the category of modal spaces where the relation is an equivalence relation. Then it is a well-known fact in modal logic that K4 is dually equivalent to TRS, S4 is dually equivalent to QOS, and S5 is dually equivalent to EQS. These results can be obtain as corollaries of our results as follows.

Let SubK $4^{\mathrm{m}}$, SubS4 ${ }^{\mathrm{m}}$, and SubS5 ${ }^{\mathrm{m}}$ be the subcategories of SubK4, SubS4, and SubS5, respectively, where morphisms are modal homomorphisms. It is then clear that SubK $4^{\mathrm{m}}$ is isomorphic to $\mathrm{K} 4, \mathrm{SubS}^{\mathrm{m}}$ is isomorphic to $\mathrm{S} 4$, and $\mathrm{SubS}^{\mathrm{m}}$ is isomorphic to S5. It is also obvious that SubK4 ${ }^{\mathrm{m}}$ is dually equivalent to TRS, SubS4 ${ }^{\mathrm{m}}$ is dually equivalent to QOS, and $\mathrm{SubS}^{\mathrm{m}}$ is dually equivalent to EQS. The duality results for $\mathrm{K} 4, \mathrm{~S} 4$, and $\mathrm{S} 5$ follow. 


\section{Lattice Subordinations and the Priestley Separation Axiom}

An interesting class of subordinations is that of lattice subordinations of [5]. In this section we show that a subordination $\prec$ on a Boolean algebra $B$ is a lattice subordination iff in the dual space $(X, R)$ of $(B, \prec)$, the relation $R$ is a Priestley quasi-order. The duality result of [5, Cor. 5.3] follows as a corollary.

Definition 5.1 A lattice subordination is a subordination $\prec$ on a Boolean algebra $B$ that in addition satisfies

$$
a \prec b \Rightarrow(\exists c \in B)(c \prec c \& a \leq c \leq b) .
$$

By [5, Lem. 2.2], a lattice subordination satisfies (S5) and (S7). In addition, since $c$ is reflexive, in the above condition, $a \leq c \leq b$ can be replaced by $a \prec c \prec b$. Therefore, a lattice subordination is a subordination that satisfies (S5) and a stronger form of (S7), where it is required that the existing $c$ is reflexive.

If $\prec$ is a lattice subordination on $B$, then as follows from the previous section, in the dual space $(X, R)$, we have that $R$ is a quasi-order. But more is true. Let $(X, R)$ be a quasiordered set. We call a subset $U$ of $X$ an $R$-upset provided $x \in U$ and $x R y$ imply $y \in U$. Similarly $U$ is an $R$-downset if $x \in U$ and $y R x$ imply $y \in U$. We recall (see, e.g., [10, 27]) that a quasi-order $R$ on a compact Hausdorff space $X$ satisfies the Priestley separation axiom if $(x, y) \notin R$ implies that there is a clopen $R$-upset $U$ such that $x \in U$ and $y \notin U$. If $R$ satisfies the Priestley separation axiom, then we call $R$ a Priestley quasi-order. Each Priestley quasi-order is closed, but the converse is not true in general $[10,30]$. A quasiordered Priestley space is a pair $(X, R)$, where $X$ is a Stone space and $R$ is a Priestley quasi-order on $X$. As was proved in [5, Cor. 5.3], lattice subordinations dually correspond to Priestley quasi-orders. To see how to derive this result from our results, we will use freely the following well-known fact about quasi-ordered Priestley spaces:

If $A, B$ are disjoint closed subsets of a quasi-ordered Priestley space $(X, R)$, with $A$ an $R$-upset and $B$ an $R$-downset, then there is a clopen $R$-upset $U$ containing $A$ and disjoint from $B$.

Lemma 5.2 Let $\prec$ be a subordination on $B$ and let $(X, R)$ be the dual of $(B, \prec)$. Then $R$ is a Priestley quasi-order iff $\prec$ satisfies (S9).

Proof First suppose that $R$ is a Priestley quasi-order. Let $a, b \in B$ with $a \prec b$. By Lemma 2.20, $R[\varphi(a)] \subseteq \varphi(b)$. Therefore, $R[\varphi(a)] \cap(X-\varphi(b))=\varnothing$. Since $R[\varphi(a)]$ is an $R$-upset, this yields $R[\varphi(a)] \cap R^{-1}[X-\varphi(b)]=\varnothing$. As $R[\varphi(a)]$ and $R^{-1}[X-\varphi(b)]$ are disjoint closed sets with $R[\varphi(a)]$ an $R$-upset and $R^{-1}[X-\varphi(b)]$ an $R$-downset, there is a clopen $R$-upset $U$ containing $R[\varphi(a)]$ and disjoint from $R^{-1}[X-\varphi(b)]$. But $U=\varphi(c)$ for some $c \in B$. Since $U$ is an $R$-upset, $R[\varphi(c)] \subseteq \varphi(c)$, so $c \prec c$. As $\varphi(a) \subseteq R[\varphi(a)] \subseteq \varphi(c)$, we have $a \leq c$. Finally, since $\varphi(c)$ is disjoint from $R^{-1}[X-\varphi(b)]$, we also have $\varphi(c) \cap(X-\varphi(b))=\varnothing$, so $\varphi(c) \subseteq \varphi(b)$, and hence $c \leq b$. Thus, $\prec$ satisfies (S9).

Next suppose that $\prec$ satisfies (S9). Then $\prec$ satisfies (S5) and (S7), hence $R$ is a quasiorder. Let $x, y \in X$ with $(x, y) \notin R$. Then $\uparrow x \nsubseteq y$. Therefore, there are $a, b \in B$ with $a \in x, a \prec b$, and $b \notin y$. By (S9), there is $c \in B$ with $c \prec c$ and $a \leq c \leq b$. From $c \prec c$ it follows that $R[\varphi(c)] \subseteq \varphi(c)$, so $\varphi(c)$ is a clopen $R$-upset of $X$. Since $a \in x$ and $a \leq c$, we have $c \in x$, so $x \in \varphi(c)$. As $c \leq b$ and $b \notin y$, we also have $c \notin y$, hence $y \notin \varphi(c)$. 
Thus, there is a clopen $R$-upset $\varphi(c)$ such that $x \in \varphi(c)$ and $y \notin \varphi(c)$, yielding that $R$ is a Priestley quasi-order.

Let LSub be the full subcategory of Sub consisting of the $(B, \prec) \in$ Sub, where $\prec$ is a lattice subordination. Let also QPS be the full subcategory of StR consisting of quasi-ordered Priestley spaces. It is an immediate consequence of our results that the dual equivalence of Sub and StR restricts to a dual equivalence of LSub and QPS. Thus, we arrive at the following result of $[5$, Cor. 5.3].

Theorem 5.3 LSub is dually equivalent to QPS.

\section{Irreducible Equivalence Relations, Compact Hausdorff Spaces, and de Vries Duality}

In this final section we prove our main results by introducing irreducible equivalence relations, Gleason spaces, and providing a "modal-like" alternative to de Vries duality. We recall [14] that a compingent algebra is a pair $(B, \prec)$, where $B$ is a Boolean algebra and $\prec$ is a binary relation on $B$ satisfying (S1)-(S8). In other words, a compingent algebra is an object of SubS5 that in addition satisfies (S8). It follows from our duality results that the dual of $(B, \prec) \in$ SubS5 is a pair $(X, R)$, where $X$ is a Stone space and $R$ is a closed equivalence relation on $X$. Since $X$ is compact Hausdorff and $R$ is a closed equivalence relation on $X$, the quotient space $X / R$ is also compact Hausdorff. In order to give the dual description of (S8), we recall that an onto continuous map $f: X \rightarrow Y$ between compact Hausdorff spaces is irreducible provided the $f$-image of each proper closed subset of $X$ is a proper subset of $Y$.

Definition 6.1 We call a closed equivalence relation $R$ on a compact Hausdorff space $X$ irreducible if the quotient map $\pi: X \rightarrow X / R$ is irreducible.

Remark 6.2 Clearly a closed equivalence relation $R$ on a compact Hausdorff space $X$ is irreducible iff for each proper closed subset $F$ of $X$, we have $R[F]$ is a proper subset of $X$. If $X$ is a Stone space, then an immediate application of Esakia's lemma $([6,19])$ yields that we can restrict the condition to proper clopen subsets of $X$.

Lemma 6.3 Let $(B, \prec) \in$ SubS5 and let $(X, R)$ be the dual of $(B, \prec)$. Then the closed equivalence relation $R$ is irreducible iff $\prec$ satisfies $(\mathrm{S} 8)$.

Proof First suppose that $R$ is irreducible. Let $a \in B$ with $a \neq 0$. Then $\varphi(a) \neq \varnothing$, so $X-\varphi(a)$ is a proper closed subset of $X$. Since $R$ is irreducible, $R[X-\varphi(a)]$ is a proper subset of $X$. Therefore, $X-R[X-\varphi(a)] \neq \varnothing$, and as $R[X-\varphi(a)]$ is closed, $X-R[X-\varphi(a)]$ is open. As $X$ is a Stone space, there is a nonempty clopen subset $U$ of $X$ contained in $X-R[X-\varphi(a)]$. But $U=\varphi(b)$ for some $b \in B$. Since $U \neq \varnothing$, we have $b \neq 0$. As $\varphi(b) \subseteq X-R[X-\varphi(a)]$ and $R$ is an equivalence relation, $R[\varphi(b)] \subseteq \varphi(a)$. Thus, there is $b \neq 0$ with $b \prec a$, and so $\prec$ satisfies (S8).

Next suppose that $\prec$ satisfies (S8). Let $F$ be a proper closed subset of $X$. Then $X-F$ is a nonempty open subset of $X$. Since $X$ is a Stone space, there is a nonempty clopen set contained in $X-F$. Therefore, there is $a \in B-\{0\}$ with $\varphi(a) \subseteq X-F$. By (S8), there is 
$b \in B-\{0\}$ with $b \prec a$. Thus, $R[\varphi(b)] \subseteq \varphi(a)$. As $R$ is an equivalence relation, this yields $\varphi(b) \subseteq X-R[X-\varphi(a)] \subseteq X-R[F]$. So $R[F] \subseteq X-\varphi(b)$. Since $b \neq 0$, we see that $X-\varphi(b)$ is a proper subset of $X$, hence $R[F]$ is a proper subset of $X$. Consequently, $R$ is irreducible.

Let Com be the full subcategory of SubS5 consisting of compingent algebras; that is, Com consists of the objects of SubS5 that in addition satisfy (S8). Let also StR ${ }^{\text {ieq }}$ be the full subcategory of $\operatorname{StR}^{\mathrm{eq}}$ consisting of the pairs $(X, R)$, where $R$ is an irreducible equivalence relation on a Stone space $X$. The above results yield:

Theorem 6.4 Com is dually equivalent to $\mathrm{StR}^{\mathrm{ieq}}$.

Definition $6.5([3,14])$ A de Vries algebra is a complete compingent algebra.

We recall that a space $X$ is extremally disconnected if the closure of every open set is open. We call an extremally disconnected Stone space an ED-space. (Equivalently, EDspaces are extremally disconnected compact Hausdorff spaces.) It is well known that a Boolean algebra $B$ is complete iff its Stone space $X$ is an ED-space. Therefore, the duals of de Vries algebras are pairs $(X, R)$, where $X$ is an ED-space and $R$ is an irreducible equivalence relation on $X$.

Definition 6.6 We call a pair $(X, R)$ a Gleason space if $X$ is an ED-space and $R$ is an irreducible equivalence relation on $X$.

Our choice of terminology is motivated by the fact that Gleason spaces arise naturally by taking Gleason covers [20] of compact Hausdorff spaces. We recall that the Gleason cover of a compact Hausdorff space $X$ is a pair $(Y, \pi)$, where $Y$ is an ED-space and $\pi: Y \rightarrow X$ is an irreducible map. It is well known that Gleason covers are unique up to homeomorphism. Suppose $X$ is compact Hausdorff and $(Y, \pi)$ is the Gleason cover of $X$. Define $R$ on $Y$ by $x R y$ iff $\pi(x)=\pi(y)$. Since $\pi$ is an irreducible map, it is easy to see that $R$ is an irreducible equivalence relation on $Y$, hence $(Y, R)$ is a Gleason space. In fact, each Gleason space arises this way because if $(Y, R)$ is a Gleason space, then as $R$ is a closed equivalence relation, the quotient space $X:=Y / R$ is compact Hausdorff. Moreover, since $R$ is irreducible, the quotient map $\pi: Y \rightarrow X$ is an irreducible map, yielding that $(Y, \pi)$ is (homeomorphic to) the Gleason cover of $X$ [20]. Thus, we have a convenient 1-1 correspondence between compact Hausdorff spaces and Gleason spaces, and both dually correspond to de Vries algebras.

Definition 6.7 ([3, 14]) A map $h: A \rightarrow B$ between two de Vries algebras is a de Vries morphism if it satisfies the following conditions:

(M1) $h(0)=0$.

(M2) $h(a \wedge b)=h(a) \wedge h(b)$.

(M3) $\quad a \prec b$ implies $\neg h(\neg a) \prec h(b)$.

(M4) $h(a)=\bigvee\{h(b): b \prec a\}$.

Remark 6.8 Condition (M3) entails a more standard condition $a \prec b$ implies $h(a) \prec h(b)$ (see [4, Lem. 2.2]) and is equivalent to $a \prec c$ and $b \prec d$ imply $h(a \vee b) \prec h(c) \vee h(d)$ [15, Prop. 3.10 and Cor. 4.20] (see also [9, Prop. 7.4]). 
It is an easy consequence of (M1) and (M3) that a de Vries morphism $h$ also satisfies $h(1)=1$. Therefore, each de Vries morphism is a meet-hemimorphism [22]. Let $X$ be the Stone space of $A$ and $Y$ be the Stone space of $B$. As follows from [22], meethemimorphisms $h: A \rightarrow B$ are dually characterized by relations $r \subseteq Y \times X$ satisfying $r[y]$ is closed for each $y \in Y$ and $r^{-1}[U]$ is clopen for each clopen $U \subseteq X$. In [22] such relations are called Boolean relations.

\section{Remark 6.9}

(1) In [22] Halmos worked with join-hemimorphisms, which generalize the modal operator $\diamond$, while meet-hemimorphisms generalize the modal operator $\square$.

(2) Boolean relations are exactly the inverses of binary JT-relations, and if $X=Y$, then Boolean relations are nothing more but Esakia relations (see Remark 4.2(2)).

We recall that the dual correspondence between $h: A \rightarrow B$ and $r \subseteq Y \times X$ is obtained as follows. Given $h: A \rightarrow B$, define $r \subseteq Y \times X$ by setting

$$
(y, x) \in r \text { iff }(\forall a \in A)(h(a) \in y \Rightarrow a \in x) .
$$

Conversely, given $r: Y \times X$, define $h: \operatorname{Clop}(X) \rightarrow \operatorname{Clop}(Y)$ by setting

$$
h(U)=Y-r^{-1}[X-U] .
$$

In order to simplify notation, instead of $(y, x) \in r$, we will often write $y r x$. We also set

$$
\square_{r} U:=Y-r^{-1}[X-U] .
$$

Thus, $h(U)=\square_{r} U$.

Definition 6.10 Suppose $r \subseteq Y \times X$.

(1) We say that $r$ is cofinal provided $(\forall y \in Y)(\exists x \in X)(y r x)$.

(2) We say that $r$ satisfies the forth condition provided

$$
\left(\forall y, y^{\prime} \in Y\right)\left(\forall x, x^{\prime} \in X\right)\left(y R y^{\prime} \& y r x \& y^{\prime} r x^{\prime} \Rightarrow x R x^{\prime}\right) .
$$

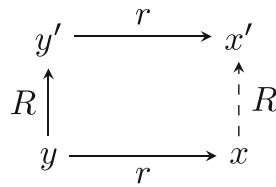

(3) We say that $r$ satisfies the de Vries condition provided

$$
(\forall U \in \operatorname{Clop}(X))\left(r^{-1}(U)=\operatorname{int}\left(r^{-1} R^{-1}[U]\right)\right) .
$$

Lemma 6.11 Let $(A, \prec)$ and $(B, \prec)$ be de Vris algebras, $(X, R)$ be the dual of $(A, \prec)$, and $(Y, R)$ be the dual of $(B, \prec)$. Suppose $h: A \rightarrow B$ is a meet-hemimorphism and $r \subseteq Y \times X$ is its dual.

(1) $h$ satisfies (M1) iff $r$ is cofinal.

(2) $h$ satisfies (M3) iff $r$ satisfies the forth condition.

(3) $h$ satisfies (M4) iff $r$ satisfies the de Vries condition.

Proof (1) We have $h(0)=0$ iff $\square_{r}(\varnothing)=\varnothing$, which happens iff $r^{-1}[X]=Y$. This in turn is equivalent to $(\forall y \in Y)(\exists x \in X)(y r x)$. Thus, $h$ satisfies (M1) iff $r$ is cofinal. 
(2) First suppose that $h$ satisfies (M3). Let $y, y^{\prime} \in Y$ and $x, x^{\prime} \in X$ with $y R y^{\prime}, y r x$, and $y^{\prime} r x^{\prime}$. To see that $x R x^{\prime}$ we must show that $\uparrow x \subseteq x^{\prime}$. Let $b \in \uparrow_{x}$. Then there is $a \in x$ with $a \prec b$. By (M3), $\neg h(\neg a) \prec h(b)$. Since $a \in x$, we have $\neg a \notin x$. As $y r x$, this yields $h(\neg a) \notin y$. Because $y$ is an ultrafilter, $\neg h(\neg a) \in y$. Therefore, $h(b) \in{ }^{\uparrow} y$. Since $y R y^{\prime}$, this gives $h(b) \in y^{\prime}$. Thus, by $y^{\prime} r x^{\prime}$, we obtain $b \in x^{\prime}$, so $x R x^{\prime}$. Consequently, $r$ satisfies the forth condition.

Next suppose that $r$ satisfies the forth condition. Let $a, b \in A$ with $a \prec b$. Then $R[\varphi(a)] \subseteq \varphi(b)$. We have $\varphi(\neg h(\neg a))=r^{-1}[\varphi(a)]$ and $\varphi(h(b))=\square_{r} \varphi(b)$. Therefore, to see that $\neg h(\neg a) \prec h(b)$, it is sufficient to show that $R\left[r^{-1}[\varphi(a)]\right] \subseteq \square_{r} \varphi(b)$. Let $y^{\prime} \in R\left[r^{-1}[\varphi(a)]\right]$. Then there are $x \in \varphi(a)$ and $y \in Y$ with $y R y^{\prime}$ and $y r x$. Suppose $x^{\prime} \in X$ with $y^{\prime} r x^{\prime}$. So $y R y^{\prime}, y r x$, and $y^{\prime} r x^{\prime}$, which by the forth condition gives $x R x^{\prime}$. Therefore, $x^{\prime} \in R[\varphi(a)]$, yielding $x^{\prime} \in \varphi(b)$. Thus, $y^{\prime} \in \square_{r} \varphi(b)$. Consequently, $R\left[r^{-1}[\varphi(a)]\right] \subseteq \square_{r} \varphi(b)$, and hence $h$ satisfies (M3).

(3) We recall that if $S \subseteq A$, then $\varphi(\bigvee S)=\overline{\bigcup\{\varphi(s): s \in S\}}$. Also, by Esakia's lemma $([6,19])$, if $\left\{U_{i}: i \in I\right\}$ is an upward directed family of clopens (meaning that for all $i, j \in I$ there is $k \in I$ such that $U_{i}, U_{j} \subseteq U_{k}$, then $\square_{r} \bigcup\left\{U_{i}: i \in I\right\}=\bigcup\left\{\square_{r} U_{i}\right.$ : $i \in I\}$. Therefore, for each $a \in A$, we have $\varphi(h(a))=\square_{r} \varphi(a)$ and

$$
\begin{aligned}
\varphi(\bigvee\{h(b): b \prec a\}) & =\overline{\bigcup\left\{\square_{r} \varphi(b): R[\varphi(b)] \subseteq \varphi(a)\right\}} \\
& =\overline{\bigcup\left\{\square_{r} \varphi(b): \varphi(b) \subseteq \square_{R} \varphi(a)\right\}} \\
& =\overline{\square_{r}\left\{\varphi(b): \varphi(b) \subseteq \square_{R} \varphi(a)\right\}} \\
& =\overline{\square_{r} \square_{R} \varphi(a)} .
\end{aligned}
$$

Thus, $h$ satisfies (M4) iff $\square_{r} \varphi(a)=\overline{\square_{r} \square_{R} \varphi(a)}$ for each $a \in A$. This is equivalent to $Y-r^{-1}[U]=Y-\operatorname{int}\left(r^{-1} R^{-1}[U]\right)$ for each $U \in \operatorname{Clop}(U)$. This in turn is equivalent to $r^{-1}[U]=\operatorname{int}\left(r^{-1} R^{-1}[U]\right)$ for each $U \in \operatorname{Clop}(U)$, yielding that $h$ satisfies (M4) iff $r$ satisfies the de Vries condition.

Definition 6.12 Let $(Y, R)$ and $(X, R)$ be Gleason spaces. We call a relation $r \subseteq Y \times X$ a de Vries relation provided $r$ is a cofinal Boolean relation satisfying the forth and de Vries conditions.

As follows from Lemma 6.11, de Vries relations dually correspond to de Vries morphisms. As with de Vries morphisms, because of the de Vries condition, the composition of two de Vries relations may not be a de Vries relation. Thus, for two de Vries relations $r_{1} \subseteq X_{1} \times X_{2}$ and $r_{2} \subseteq X_{2} \times X_{3}$, we define $r_{2} * r_{1} \subseteq X_{1} \times X_{3}$ as follows. Let $h_{1}: \operatorname{Clop}\left(X_{2}\right) \rightarrow \operatorname{Clop}\left(X_{1}\right)$ be the dual of $r_{1}$ and $h_{2}: \operatorname{Clop}\left(X_{3}\right) \rightarrow \operatorname{Clop}\left(X_{2}\right)$ be the dual of $r_{2}$. Let $h_{3}=h_{1} * h_{2}$ be the composition of $h_{1}$ and $h_{2}$ in the category DeV of de Vries algebras. Then $h_{3}: \operatorname{Clop}\left(X_{3}\right) \rightarrow \operatorname{Clop}\left(X_{1}\right)$ is a de Vries morphism. Let $r_{3} \subseteq X_{1} \times X_{3}$ be the dual of $h_{3}$, and set $r_{3}=r_{2} * r_{1}$. With this composition, Gleason spaces and de Vries relations form a category we denote by Gle. We also let KHaus denote the category of compact Hausdorff spaces and continuous maps. The following is an immediate consequence of the above observations.

Theorem 6.13 Gle is dually equivalent to $\mathrm{DeV}$, hence Gle is equivalent to KHaus. 
Thus, Gle is another dual category to DeV. This provides an alternative more "modallike" duality to de Vries duality.

Remark 6.14 The functor $\Phi:$ Gle $\rightarrow$ KHaus establishing an equivalence of Gle and KHaus can be constructed directly, without first passing to DeV. For $(X, R) \in$ Gle, let $\Phi(X, R)=X / R$. Clearly $X / R \in$ KHaus. For $r \subseteq Y \times X$ a morphism in Gle, let $\Phi(r)=f$, where $f: Y / R \rightarrow X / R$ is defined as follows. Let $\pi: X \rightarrow X / R$ be the quotient map. Since $r$ is cofinal, for each $y \in Y$ there is $x \in X$ with $y r x$. We set $f(\pi(y))=\pi(x)$, where $y r x$. Since $r$ satisfies the forth condition, $f$ is well defined, and as $r$ is a Boolean relation, $f$ is continuous. Thus, $f$ is a morphism in KHaus. From this it is easy to see that $\Phi$ is a functor. We already saw that there is a 1-1 correspondence between Gleason spaces and compact Hausdorff spaces. The functor $\Phi$ is full because for each continuous function $f: Y \rightarrow X$ between compact Hausdorff spaces, $f=\Phi(r)$, where $r$ is the de Vries relation corresponding to the de Vries dual of $f$. Finally, the functor is faithful because among the cofinal Boolean relations $r$ that satisfy the forth condition and yield the same continuous function $f: Y \rightarrow X$ in KHaus, there is the largest one, which satisfies the de Vries condition. Consequently, by [25, Thm. IV.4.1], $\Phi:$ Gle $\rightarrow$ KHaus is an equivalence.

Open Access This article is distributed under the terms of the Creative Commons Attribution 4.0 International License (http://creativecommons.org/licenses/by/4.0/), which permits unrestricted use, distribution, and reproduction in any medium, provided you give appropriate credit to the original author(s) and the source, provide a link to the Creative Commons license, and indicate if changes were made.

\section{References}

1. Aleksandrov, P.S., Ponomarev, V.I.: Compact extensions of topological spaces (Russian). Vestnik Moskov. Univ. Ser. Mat. Meh. Astr. Fiz. Him. 5, 93-108 (1959)

2. Balbiani, P., Kikot, S.: Sahlqvist theorems for precontact logics. In: Advances in Modal Logic 9, Papers from the Ninth Conference on "Advances in Modal Logic," Held in Copenhagen, Denmark, 22-25 August 2012, pp. 55-70 (2012)

3. Bezhanishvili, G.: Stone duality and Gleason covers through de Vries duality. Topol. Appl. 157(6), 1064-1080 (2010)

4. Bezhanishvili, G.: De Vries algebras and compact regular frames. Appl. Categ. Struct. 20(6), 569-582 (2012)

5. Bezhanishvili, G.: Lattice subordinations and Priestley duality. Algebra Univers. 70(4), 359-377 (2013)

6. Bezhanishvili, G., Bezhanishvili, N., Harding, J.: Modal compact Hausdorff spaces. J. Logic Comput. 80(2), 520-566 (2015)

7. Bezhanishvili, G., Bezhanishvili, N., Iemhoff, R.: Stable canonical rules. J. Symb. Log. 81(01), 284315 (2016)

8. Bezhanishvili, G., Bezhanishvili, N., Sourabh, S., Venema, Y.: Subordinations, closed relations, and compact Hausdorff spaces. ILLC Prepublication Series, PP-2014-23. Available at http://www.illc.uva. nl/Research/Publications/Reports/PP-2014-23.text.pdf (2014)

9. Bezhanishvili, G., Harding, J.: Proximity frames and regularization. Appl. Categ. Struct. 81(01), 284315 (2016)

10. Bezhanishvili, G., Mines, R., Morandi, P.J.: The Priestley separation axiom for scattered spaces. Order 19(1), 1-10 (2002)

11. Blackburn, P., de Rijke, M., Venema, Y.: Modal Logic. Cambridge University Press, Cambridge (2001)

12. Chagrov, A., Zakharyaschev, M.: Modal Logic, volume 35 of Oxford Logic Guides. The Clarendon Press, New York (1997)

13. Celani, S.: Quasi-modal algebras. Math. Bohem. 126(4), 721-736 (2001)

14. de Vries, H.: Compact spaces and compactifications. An algebraic approach. $\mathrm{PhD}$ thesis, University of Amsterdam (1962)

15. Dimov, G.: A de Vries-type duality theorem for the category of locally compact spaces and continuous maps. I. Acta Math. Hungar. 129(4), 314-349 (2010) 
16. Dimov, G., Vakarelov, D.: Topological representation of precontact algebras. In: W. MacCaull, M. Winter, I. Düntsch (eds.) Relational Methods in Computer Science, volume 3929 of Lecture Notes in Computer Science, pp. 1-16. Springer, Berlin (2006)

17. Dimov, G., Vakarelov, D.: Topological representation of precontact algebras and a connected version of the Stone duality theorem-I. Available at http://arxiv.org/pdf/1508.02220v2.pdf (2015)

18. Düntsch, I., Vakarelov, D.: Region-based theory of discrete spaces: a proximity approach. Ann. Math. Artif. Intell. 49(1-4), 5-14 (2007)

19. Esakia, L.: Topological Kripke models. Soviet Math. Dokl. 15, 147-151 (1974)

20. Gleason, A.M.: Projective topological spaces. Ill. J. Math. 2, 482-489 (1958)

21. Goldblatt, R.: Varieties of complex algebras. Ann. Pure Appl. Logic 44(3), 173-242 (1989)

22. Halmos, P.R.: Algebraic Logic. Chelsea Publishing Co., New York (1962)

23. Jónsson, B., Tarski, A.: Boolean algebras with operators. I. Am. J. Math. 73, 891-939 (1951)

24. Kracht, M.: Tools and Techniques in Modal Logic. North-Holland Publishing Co., Amsterdam (1999)

25. Mac Lane, S.: Categories for the Working Mathematician, 2nd edn. Springer, New York (1998)

26. Naimpally, S.A., Warrack, B.D.: Proximity Spaces. Cambridge Tracts in Mathematics and Mathematical Physics, No. 59. Cambridge University Press, London (1970)

27. Priestley, H.A.: Ordered topological spaces and the representation of distributive lattices. Proc. Lond. Math. Soc. 24, 507-530 (1972)

28. Sofronie-Stokkermans, V.: Duality and canonical extensions of bounded distributive lattices with operators, and applications to the semantics of non-classical logics I. Stud. Log. 64(1), 93-132 (2000)

29. Stone, M.H.: The theory of representations for Boolean algebras. Trans. Am. Math. Soc. 40(1), 37-111 (1936)

30. Stralka, A.: A partially ordered space which is not a Priestley space. Semigroup Forum 20(4), 293-297 (1980) 